\title{
Multi-epoch spectroscopy, photometry and polarimetry of the polar UW Pic
}

\author{
E. Romero-Colmenero, S.B. Potter, D.A.H. Buckley
}

South African Astronomical Observatory, P.O. Box 9, Observatory, 7935, South Africa

\section{P.E. Barrett}

Space Telescope Science Institute, ESS/SSG, 3700 San Martin Drive, Baltimore, MD 21218

\section{S. Vrielmann}

Department of Astronomy, University of Cape Town, Private Bag, Rondebosch, 7700, South Africa

\begin{abstract}
Our multi-epoch observations show that UW Pic undergoes very pronounced changes in accretion geometry. We explain our observations in terms of changing accretion rates of UW Pic coupled with a particular orientation of the system to the observer.
\end{abstract}

We have taken photometric, polarimetric and spectroscopic observations of the ROSAT source RX J0531.5 - 4624 (UW Pic), previously identified as a polar, over the period of about a decade. Our observations show that UW Pic undergoes very pronounced changes in accretion geometry; this is particularly evident from the polarimetric observations. At times, UW Pic shows low levels of both positive and negative circular polarization, suggesting simultaneous accretion onto two magnetic poles. At other times, UW Pic shows high levels of negative circular polarization only over the whole orbit, suggesting accretion onto one dominant pole. Our photometric measurements also show morphological variations on monthly timescales. The HeII ( $\lambda 4686)$ emission line varies in strength between epochs, being comparable to $\mathrm{H} \beta$ at one epoch and almost non-existent at others. Similarly, CaII ( $\lambda$ 3933) can either be weak or absent, its strength appearing to be in anti-correlation with that of HeII $(\lambda 4686)$.
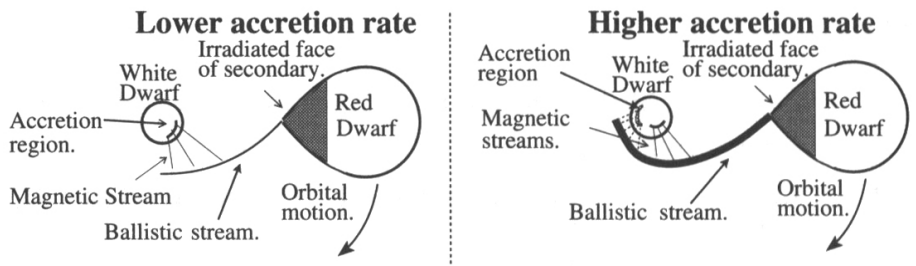

Figure 1. Possible scenarios for different accretion rates in UW Pic. 

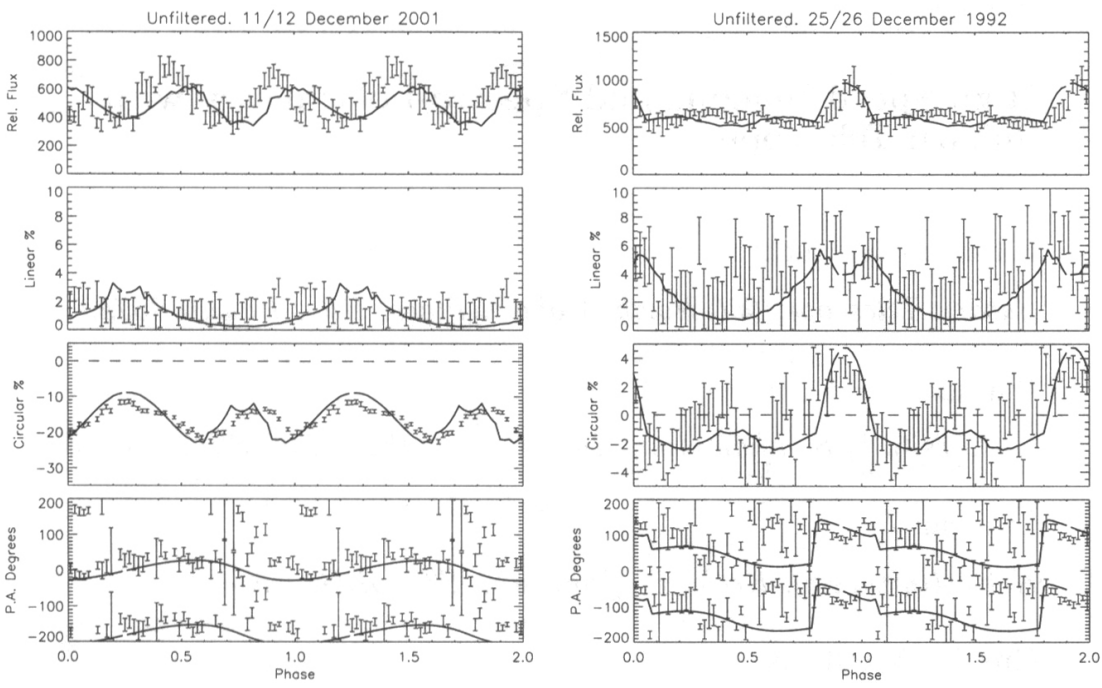

Figure 2. Left:One pole model (solid line) over-plotted on the polarimetry data taken on 11/12 December 2001. Right: Two pole model (solid line) over-plotted on the polarimetry data taken on 25/26 December 1992.

We put forward a scenario that explains our observations in terms of changing accretion rates of UW Pic coupled with a particular orientation of the system to the observer (see Figure 1).

We have also modelled the polarimetric observations using two models, each representing the two general states of accretion.

The high accretion rate model lightcurve was created by arbitrarily placing an emission spot at $\theta=18^{\circ}$ below the magnetic pole, in the hemisphere of the white dwarf most pointing towards the observer (the upper pole). The results can be seen in Figure 2: this model has reproduced the double humped morphology of the intensity and negative circular polarization quite well.

For the low accretion rate model, the same emission spot we used previously was again placed in the upper hemisphere. However, this time we added a second accretion spot located $18^{\circ}$ from the opposite magnetic pole in the lower hemisphere of the white dwarf. The results are also shown in Figure 2: this model has again reproduced the main features of the lightcurve quite well.

Full details of this work are presented in Romero-Colmenero et al. (2003).

\section{References}

Romero-Colenero, E., Potter, S. B., Buckley, D. A. H., Barrett, P.E. \& Vrielmann, S., 2003, MNRAS, 339, 685 